\title{
Comportamiento de los nutrientes en tejido foliar en brócoli (Brassica oleracea var. italica) 'Coronado' y repollo (Brassica oleracea) híbrido 'Delus' cultivados en la Sabana de Bogotá
}

\section{Nutrient levels in foliar tissue of broccoli (Brassica oleracea var. italica) 'Coronado' and cabbage (Brassica oleracea) hybrid 'Delus' cultivated on the Bogota Plateau}

CARLOS CARRANZA ${ }^{1}$

OCTAVIO LANCHERO

DIEGO MIRANDA 1,2

Brócoli en el Centro Agropecuario Marengo, Mosquera, Bogotá.

Foto: C. Carranza

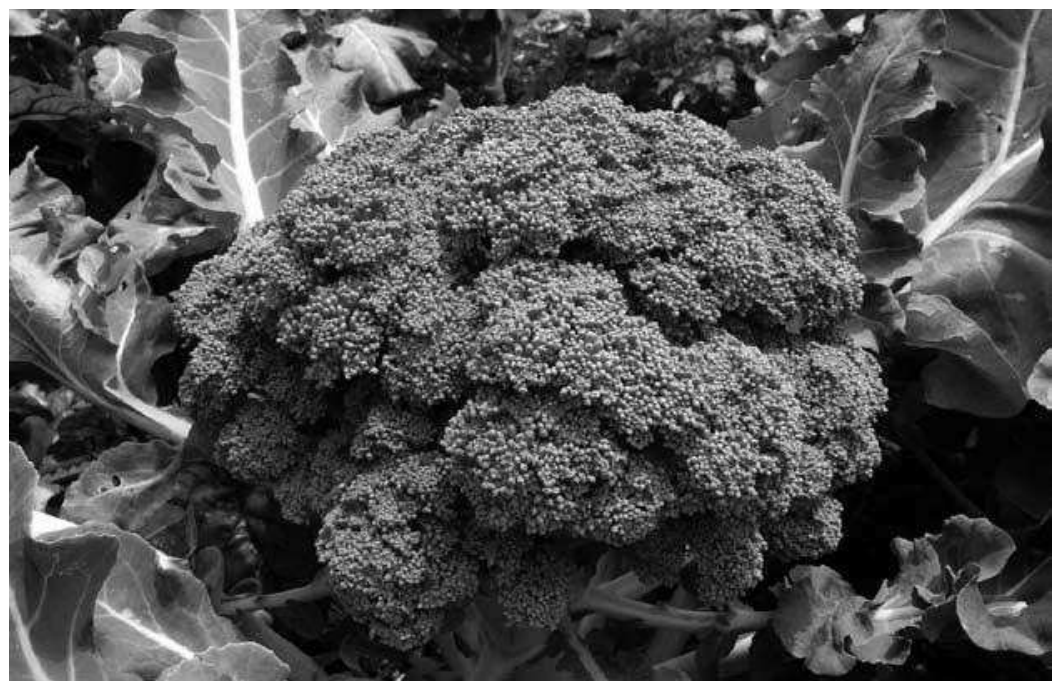

\section{RESUMEN}

De las Brassicas más importantes que se cultivan en la Sábana de Bogotá son el brócoli y el repollo, pero no existe información de los nutrientes en estrés salino. En el centro agropecuario Marengo de la Universidad Nacional de Colombia, en tres parcelas de $300 \mathrm{~m}^{2}$ cada una, con 8,3 y 11,1 plantas $/ \mathrm{m}^{2}$ para brócoli y repollo respectivamente, regadas con aguas del distrito La Ramada, se realizaron muestreos cada ocho días y se determinó el contenido total de nutrientes N, P, K, Ca, Mg y B en el tejido foliar. Los datos obtenidos se ajustaron a curvas cuadráticas. En brócoli, los contenidos de N, P, Ca y Mg estuvieron entre los rangos adecuados los primeros 51 días después del trasplante (ddt) y posteriormente fueron deficientes. En repollo el $\mathrm{P}, \mathrm{Ca}$ y $\mathrm{Mg}$ se ubicaron en los rangos adecuados. El $\mathrm{K}$ fue deficiente en todo el ciclo del cultivo para brócoli y repollo. El contenido del B fue alto en brócoli a los $19 \mathrm{ddt}$ y en repollo a los $47 \mathrm{ddt}$. De acuerdo con la respuesta de las plantas, la presencia de sales tanto en el suelo como en el agua de riego influyó en la absorción, transporte distribución de los elementos minerales en las especies de Brassica evaluadas.

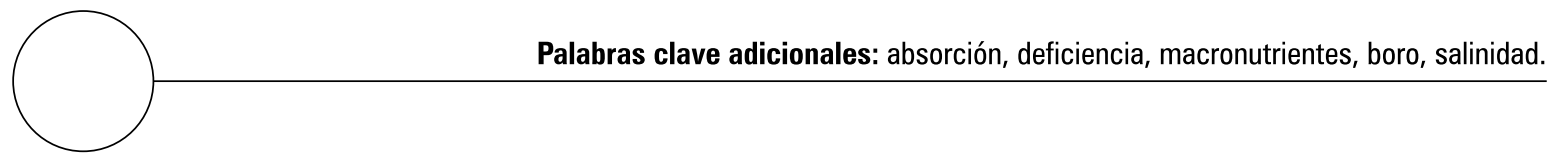

Departamento de Agronomía, Facultad de Agronomía, Universidad Nacional de Colombia, Bogotá.

Autor para correspondencia.dmirandal@unal.edu.co 


\section{ABSTRACT}

The objective of this study was to evaluate changes in plant nutrient levels over time in broccoli and cabbage crops grown in the open field on the Bogota Plateau. The study was conducted at Agricultural Center Marengo of the National University of Colombia located at $\mathrm{km} 12$ route Bogota-Mosquera, 2,516 $\mathrm{m}$ altitude above sea level, and characterized by an average temperature of $13.7^{\circ} \mathrm{C}$, precipitation $669.9 \mathrm{~mm}$ year ${ }^{-1}$ and 4.2 daily sunshine hours, in a moderately saline soil. Three plots of $300 \mathrm{~m}^{2}$ each, with respectively 8.3 and 11.1 plants per $\mathrm{m}^{2}$ of broccoli and cabbage were irrigated with water of La Ramada district. Samples were taken every 8 days and the content of the plant nutrients $\mathrm{N}, \mathrm{P}, \mathrm{K}, \mathrm{Ca}, \mathrm{Mg}$ and $\mathrm{B}$ in foliar tissue was determined. Data generally fit a quadratic curve over time. In broccoli, the contents of $\mathrm{N}, \mathrm{P}, \mathrm{Ca}$ and $\mathrm{Mg}$ remained within an adequate range during the first 51 days after the transplanting (dat) but later became deficient. In cabbage, the contents of $\mathrm{P}, \mathrm{Ca}$ and $\mathrm{Mg}$ were found to be within suitable ranges. Potassium was deficient during the whole crop cycle in both broccoli and cabbage. Boron was high in broccoli until 19 dat and, in cabbage, until 47 dat. In accordance with plant response, the presence of salts both in the soil and irrigation water probably influenced the absorption, transport and distribution of mineral elements in evaluated species of Brassicaceae.

Additional keywords: absorption, deficiency, macronutrients, boron, salinity.

Fecha de recepción: 19-12-2007

Aprobado para publicación: 03-06-2008

INTRODUCCIÓN

La producción agrícola mundial está ampliamente afectada por un sinnúmero de peligros ambientales, entre los cuales la salinidad es uno de los más importantes y está asociado con la sequía. Las tierras salinas no solamente están distribuidas en zonas desérticas o semidesérticas, también ocurren frecuentemente en suelos planos, fértiles, valles y regiones costeras densamente pobladas y con sistemas de irrigación (Sen et al., 2002). De 230 millones de hectáreas irrigadas, 45 millones han sido afectadas por sales (19,5\%) y de 1.500 millones de hectáreas bajo agricultura de "tierras secas", 32 millones están afectadas por sales en diferentes grados (Munns, 2003).

En la clasificación americana de suelos USDA (2006) Soil Taxonomy, se estableció que, a partir del valor de $2 \mathrm{dS} \mathrm{m}^{-1}$, el suelo se considera salino y las propiedades morfológicas y fisicoquímicas del perfil quedan influenciadas por el carácter salino. Por otra parte, el laboratorio de salinidad de los Estados Unidos estableció como límite 4 $\mathrm{d} S \mathrm{~m}^{-1}$ para que la salinidad comience a ser tóxica para las plantas (Greenway y Munns, 1980; Marschner, 2002). Existe también un espectro continuo de tolerancia de plantas a condiciones salinas clasificándolas como glicófitas, que son sensibles a la sal hasta las halófitas, las cuales sobreviven en concentraciones muy altas de sales (Volkmar et al., 1998).

El estrés por salinidad es un fenómeno complejo e involucra no sólo el efecto osmótico, sino también el efecto de iones tóxicos y el desbalance nutricional (Ashraf, 2004). El factor iónico de la salinidad radica en la toxicidad iónica. Los iones que más problemas inducen son el cloruro $\left(\mathrm{Cl}^{-}\right)$ y el sodio $\left(\mathrm{Na}^{+}\right)$, aunque otros, como el nitrato $\left(\mathrm{NO}_{3}^{-}\right)$, el sulfato $\left(\mathrm{SO}_{4}^{2-}\right)$ el amonio $\left(\mathrm{NH}_{4}^{+}\right)$o el borato $\left(\mathrm{H}_{2} \mathrm{BO}_{3}^{-2}\right)$ también pueden ser tóxicos. Su acumulación en las hojas reduce la tasa fotosintética, induce la pérdida de pigmentos y provoca la senescencia y la abscisión (Azcón-Bieto y Talón, 2008). 
El brócoli (Brassica oleracea var. italica) y repollo (Brassica oleracea) pertenecen a la familia Brassicaceae y según la Encuesta Nacional Agropecuaria realizada por el Ministerio de Agricultura y Desarrollo Rural en 2006, en Colombia se sembraron 2.186 ha, donde se cosecharon 2.150 ha para un volumen total de 50.767 t (CCI, 2006) y para 2007 en la Sabana de Bogotá, se sembraron 500 ha con una producción anual de 14.457 t (Asohofrucol, 2007). Otras zonas productoras incluyen sectores de Cundinamarca, Boyacá, Antioquia, Nariño, Tolima y Caldas (Semillas Arroyave, 2007). El híbrido Delus es semi-tardío, se cosecha entre 115 y 120 días después del trasplante, tiene buena tolerancia a bajas temperaturas y es medianamente resistente a la salinidad (Semillas Arroyave, 2007).

A pesar de que el brócoli y el repollo son de las Brassicas más importantes que se cultivan en la Sabana de Bogotá, no existe información acerca del comportamiento de los nutrientes cuando la planta está en condiciones de estrés salino. Por lo anterior, esta investigación busca determinar el comportamiento de los contenidos de nutrientes en el tejido foliar de brócoli y repollo cultivados en un suelo salino de la Sabana de Bogotá.

\section{MATERIALES Y MÉTODOS}

El ensayo se realizó en el Centro Agropecuario Marengo de la Universidad Nacional de Colombia, sede Bogotá, ubicado en el km 12 vía Bogotá-Mosquera, con coordenadas $4^{\circ} 42^{\prime} \mathrm{N}$; $74^{\circ} 12^{\prime}$ $\mathrm{W}$, a $2.543 \mathrm{msnm}$, temperatura anual $12,6^{\circ} \mathrm{C}$, precipitación $669,9 \mathrm{~mm}$ año ${ }^{-1}$ y brillo solar $4,2 \mathrm{~h}$ diarias. Para cada especie, el diseño experimental consistió en tres repeticiones, con parcelas de 300 $\mathrm{m}^{2}$ por repetición, con la siembra de plántulas de brócoli variedad 'Coronado' a una densidad de 8,3 plantas $/ \mathrm{m}^{2}$ y para repollo híbrido 'Delus' en una densidad de 11,1 plantas $/ \mathrm{m}^{2}$.

La fertilización se efectuó a partir de los resultados del análisis de suelo (tabla 1) realizado en el Laboratorio de Suelos y Aguas, Facultad de Agronomía, Universidad Nacional de Colombia. Se emplearon los siguientes métodos: carbono orgánico (CO): método de Walkley-Black; N: estimado a partir del CO (factor empleado: 0,0862); Ca, K, Mg, Na intercambiables: extracción con acetato de amonio $1 \mathrm{~N} \mathrm{pH} \mathrm{7,} \mathrm{valoración} \mathrm{por} \mathrm{absorción} \mathrm{atómica;} \mathrm{CIC}$ (Capacidad de Intercambio Catiónico): desplazamiento del $\mathrm{NH}_{4}$ intercambiado con $\mathrm{NaCl} 1 \mathrm{M}$, valoración volumétrica; $\mathrm{P}$ aprovechable: método de Bray II, valoración colorimétrica; $\mathrm{Cu}, \mathrm{Fe}, \mathrm{Mn}, \mathrm{Zn}$ : extracción con DTPA, valoración por absorción atómica; B: extracción con fosfato monobásico (Azometina-H) (ICA, 1989; IGAC, 1990), valoración colorimétrica; Arcilla (Ar), limo (L), arena (A): método de Bouyoucos, previa dispersión con hexametafosfato de sodio; textura: triángulo de clasificación textural del USDA. Los nutrientes en deficiencia se suplementaron con fertilizantes químicos edáficos de mayores N 15\%, P 30\% y K $10 \%$, y menores $\mathrm{N} 8,0 \%$ (Amoniacal 1,0\% y Ureico 7,0\%); $\mathrm{P}_{2} \mathrm{O}_{5} 5,0 \%$; $\left.\mathrm{CaO} 18,0 \% ; \mathrm{MgO}\right) 6,0 \% ; \mathrm{S}$ 1,6\%; B 1,0\%; Cu 0,75\%; Mo 0,005\%; Zn 2,5. En mezcla 1:1 con una dosis de $10 \mathrm{~g} /$ planta $^{-1}$, los primeros 30 días, y posteriormente dos aplicaciones 45 y 70 días después del trasplante con N 15\%, P $15 \%$ y $\mathrm{K} 15 \%$ en dosis de $20 \mathrm{~g} /$ planta. El suelo presentó una conductividad eléctrica de $2,16 \mathrm{dS} \mathrm{m}^{-1} \mathrm{y}$ el agua de riego $0,95 \mathrm{dS} \mathrm{m}^{-1}$. El manejo agronómico de malezas, plagas y enfermedades se efectuó oportunamente, dependiendo de las poblaciones, niveles y síntomas; finalmente, no se presentaron problemas relevantes que afectaran el desarrollo de las plantas.

Tabla 1. Características fisicoquímicas del suelo, Centro Agropecuario Marengo, Universidad Nacional de Colombia.

\begin{tabular}{|c|c|c|c|c|c|c|c|c|c|c|c|c|c|c|c|c|c|}
\hline \multirow{2}{*}{ pH } & $\mathrm{CO}$ & $\mathrm{N}$ & $\mathrm{Ca}$ & K & $\mathrm{Mg}$ & $\mathrm{Na}$ & $\mathrm{CIC}$ & $P$ & $\mathrm{Cu}$ & $\mathrm{Fe}$ & $\mathrm{Mn}$ & $\mathrm{Zn}$ & B & $\mathrm{Ar}$ & L & A & \\
\hline & \multicolumn{2}{|c|}{$\%$} & \multicolumn{5}{|c|}{ meq $100 \mathrm{~g}^{-1}$} & \multicolumn{6}{|c|}{$\mathrm{mg} \mathrm{kg}^{-1}$} & \multicolumn{3}{|c|}{$\%$} & \\
\hline 5,59 & 4,08 & 0,35 & 10,5 & 0,46 & 3,51 & 4,94 & 27,8 & 48,1 & 1,04 & 426 & 1,49 & 21,8 & 0,94 & 17 & 47 & 36 & $\mathrm{Fr}$ \\
\hline
\end{tabular}


Los muestreos se realizaron cada ocho días en tres plantas por parcela y por repetición, para un total de siete muestreos en brócoli y ocho en repollo. En el Laboratorio de Agua y Suelos de la Facultad de Agronomía, Universidad Nacional de Colombia, se determinó el contenido total de nutrientes en el tejido foliar de acuerdo con las metodologías del ICA (1989) e IGAC (1990). N (nitrógeno total): método de micro-Kjeldahl, valoración volumétrica; P: calcinación de la muestra, valoración calorimétrica con vanadato y molibdato de amonio; Ca, K, Mg: calcinación de la muestra, valoración por espectrofotometría de absorción atómica; B: calcinación de la muestra, valoración colorimétrica con Azometina-H. Los datos obtenidos fueron ajustados a una regresión cuadrática de la forma:

$Y=a x^{2}+b x+c$

En la cual:

Y: la concentración del elemento

$\mathrm{x}$ : días después del trasplante

a, b y c: coeficientes del modelo

\section{RESULTADOS Y DISCUSIÓN}

Los cultivos pueden ser adversamente afectados por la salinidad, induciendo desórdenes nutricionales. Estos desórdenes pueden resultar de los efectos de la salinidad sobre la disponibilidad de nutrientes, respuesta competitiva, transporte o partición dentro de la planta (Grattan y Grieve, 1999). El contenido nutricional de las Brassicas es variable y depende de las condiciones ambientales en las que se desarrolla la planta, la edad de la misma y manejo del cultivo. En nuestro estudio, la concentración de nitrógeno total en el tejido foliar de brócoli presentó un mayor porcentaje $(5,78 \%)$ al inicio del cultivo, encontrándose en los rangos óptimos (4,0-6,5\%) (Castellanos, 1998), y luego disminuyó a través del ciclo pro- ductivo hasta el momento de la cosecha (2,78\%), llegando al rango de deficiencia en las hojas (figura 1A). En repollo, los rangos óptimos para el $\mathrm{N}$ en tejido foliar varían entre $3 \%$ y $4 \%$, considerados niveles adecuados para el tejido foliar (Marambio, 2004). En nuestro caso, la concentración obtenida fue alta $(5,97 \%)$ en los muestreos iníciales y disminuyó progresivamente hasta los 68 días después del trasplante (ddt) (2,71\%) (figura 2A). Por tanto, en los primeros $53 \mathrm{ddt}$ el contenido de $\mathrm{N}$ estuvo en el rango óptimo y de ahí hasta el final del ciclo productivo estuvo en el rango de deficiencia. Este comportamiento fue investigado por Greenway y Munns (1980), quienes aseguraron que la salinidad en muchos suelos incrementa el contenido de $\mathrm{Na} \mathrm{y}$, consecuentemente, afecta la absorción de otros elementos minerales. Es posible que las disminuciones en el contenido de $\mathrm{N}$ en hojas también estén asociadas al alto consumo del $\mathrm{N}$ en las etapas intermedias del desarrollo del cultivo.

Las relaciones salinidad-nitrógeno en las especies hortícolas son muy complejas. Sin embargo, la mayoría de estudios reportan que la toma y acumulación de $\mathrm{N}$ en el tallo de las plantas puede ser reducido bajo condiciones de salinidad, aunque otros estudios han encontrado respuestas contrarias, o simplemente no han encontrado efecto, como lo menciona (Feigin, 1985). El efecto de la salinidad sobre el consumo de nitratos varía considerablemente con la especie y las condiciones experimentales. En especies del Ricinus, la salinidad tiene efecto para asignar la reducción de nitratos en una alta proporción en las raíces (Peuke et al., 1996). Cramer et al. (1995) concluyen que el consumo en la raíces de $\mathrm{NO}_{3}{ }^{-}$ en solución es inhibida por la salinización; consecuentemente, las concentraciones de $\mathrm{NO}_{3}^{-}$en las hojas y tallos con alta actividad del nitrato reductasa es reducida por la salinidad, en comparación con las plantas sin tratamiento salino.

En brócoli, la concentración de fósforo en el tejido foliar fue alta $(0,54 \%)$ al inicio del cultivo y disminuyó a $0,39 \%$; el rango óptimo para 
este nutriente varía entre 0,45 y $0,8 \%$; en los primeros $51 \mathrm{ddt}$, el contenido de $\mathrm{P}$ estuvo entre los rangos óptimos sugeridos por Castellanos (1998) (figura 1B). En repollo, el comportamiento de la concentración de $\mathrm{P}$ (figura $2 \mathrm{~B}$ ) fue alto a los $19 \mathrm{ddt}(0,54 \%)$, disminuyó hasta los $47 \mathrm{ddt}$ $(0,42 \%)$ y nuevamente aumentó hasta los $68 \mathrm{ddt}$ $(0,48 \%)$. El rango óptimo de $\mathrm{P}$ en tejido foliar es de $0,4-0,7 \%$ (Marambio, 2004), por consiguiente la concentración del $\mathrm{P}$ estuvo entre los rangos óptimos. El P es un constituyente de ácidos nucleicos, fosfolípidos, fosfoproteínas, dinucleótidos y adenosin trifosfato. De ahí que es requerido para diferentes procesos, incluyendo almacenamiento y transferencia de energía, fotosíntesis, la regulación de enzimas y el transporte de carbohidratos (Hu y Schmidhalter, 2005). Papadopoulous y Rendig (1983), Sharpley et al. (1992), Grattan y Grieve (1999) reportan que disminuciones en el P total en el tejido de las plantas tienden a estar relacionadas con incrementos en la salinidad y, en particular, con las concentraciones de $\mathrm{Ca}^{2+}$ en el sustrato. Esto puede ser atribuido a la formación de fosfatos de $\mathrm{Ca}^{2+}$, así la concentración de $\mathrm{Ca}^{2+}$ se incrementa en el suelo haciéndolo no disponible para la planta. Patel y Pandey (2007) encontraron en plántulas de Cassia montana que el $\mathrm{P}$ y Ca ${ }^{2+}$ son factores limitantes para el cre-

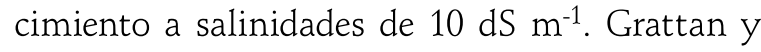
Grieve (1999) mencionan que en suelos salinos la disponibilidad del fosfato es reducida principalmente por procesos de absorción y por la baja solubilidad de los minerales de $\mathrm{Ca}^{2+}$ y $\mathrm{P}$.

El potasio es un elemento esencial para la síntesis de proteínas, enzimas glicolíticas y fotosíntesis, un potencial osmótico durante la expansión celular y turgor; también es un competidor de $\mathrm{Na}^{+}$ bajo condiciones salinas (Marschner, 2002). En brócoli, el comportamiento del $\mathrm{K}^{+}$en tejido foliar presentó una alta concentración en el muestreo inicial (2,94\%), disminuyó progresivamente hasta los $51 \mathrm{ddt}(1,95 \%)$ y aumentó levemente hasta los $61 \mathrm{ddt}(2,06 \%)$. Es posible que esta disminución en los contenidos de $\mathrm{K}^{+}$esté asociada con el efecto competitivo con el $\mathrm{Na}^{+}$, pero también con el hecho conocido de que esta especie es altamente consumidora de $\mathrm{K}^{+}$en las etapas intermedias del cultivo. La concentración ideal de $\mathrm{K}^{+}$en el tejido foliar se ubica en el rango de 3,5-6,0\% (Castellanos, 1998). De acuerdo con nuestros resultados, se presentaría una deficiencia marcada en la concentración de este elemento en el tejido foliar (figura 1C). En repollo, la concentración de $\mathrm{K}^{+}$en el tejido foliar (figura 2C) a los $19 \mathrm{ddt}$ fue 2,2\% y aumentó progresivamente a los $68 \mathrm{ddt}$ hasta $3,23 \%$, siendo éste elemento deficiente durante todo el ensayo, ya que el rango óptimo de $\mathrm{K}^{+}$ se encuentra entre 4,5 y 7,5\% (Marambio, 2004). $\mathrm{El} \mathrm{K}^{+}$es el soluto inorgánico más importante en plantas y como tal hace una mayor contribución al bajo potencial osmótico en la estela de la raíz, que es un indispensable prerrequisito para conducir la presión de turgor en el transporte de solutos en el xilema y el balance hídrico en las plantas (Marschner, 2002). La deficiencia de $\mathrm{K}^{+}$ inducida por $\mathrm{Na}^{+}$está implicada en la reducción del crecimiento y rendimiento de varios cultivos, incluyendo tomate (López y Satti, 1996; Song y Fujiyama, 1996), espinaca (Chow et al., 1990), hinojo (Graifenberg et al., 1996), y maíz (Botella et al., 1997). La reducción en el consumo de $\mathrm{K}^{+}$ en plantas por $\mathrm{Na}^{+}$es un proceso competitivo y ocurre aún a pesar de todo si la solución es dominada por sales de $\mathrm{Na}^{+}$, de $\mathrm{Cl}^{-}$o de $\mathrm{SO}_{4}{ }^{2-}$

En brócoli, la concentración de calcio en tejido foliar indica un alto porcentaje $(2,44 \%)$ al inicio del cultivo, disminuyendo progresivamente hasta los 47 días $(0,98 \%)$ y aumentando levemente hasta el momento de la cosecha (1,37\%). En los primeros $23 \mathrm{ddt}$, la concentración de $\mathrm{Ca}^{2+}$ total en la hoja se encontró en el rango óptimo (2-4\%) (Castellanos, 1998), mientras que al final del cultivo estuvo en un rango de deficiencia, indicando cierta influencia de la salinidad del suelo en la absorción de $\mathrm{Ca}^{2+}$ por parte de la planta (figura 1D). En repollo, la concentración de $\mathrm{Ca}^{2+}$ (figura 2D) en el tejido foliar fue alto a los $19 \mathrm{ddt}(2,6 \%)$ y disminuyó hasta la cosecha a los 68 días con $1,07 \%$, valores considerados ligeramente por debajo del rango de suficiencia $(1,9-6,0 \%)$ determi- 
nado por Marambio (2004). El Ca ${ }^{2+}$ desempeña un papel esencial en la integridad estructural y funcional de las membranas de las plantas, estabilidad de la pared celular, regulación de transporte de iones y permeabilidad de membranas, control del intercambio iónico, así como la actividad enzimática de la pared celular (Rengel, 1992; Marschner, 2002). En las plantas, el $\mathrm{Na}^{+}$ puede desplazar el $\mathrm{Ca}^{2+}$ de las membranas celulares, modificando la absorción de nutrientes, como el $\mathrm{K}^{+}$. Sin embargo, se ha observado que si existe un aporte suplementario de $\mathrm{Ca}^{2+}$ en el proceso de absorción de $\mathrm{K}^{+}$funciona bien, en detrimento del $\mathrm{Na}^{+}$. Así, la fertilización con $\mathrm{Ca}^{2+}$ mejora la producción agrícola al incrementar la absorción de $\mathrm{K}^{+}$y reducir el flujo de $\mathrm{NO}_{3}, \mathrm{PO}_{4}$ (Azcón-Bieto y Talón, 2008). Concentraciones adecuadas de $\mathrm{Ca}^{2+}$ pueden tener un papel importante en la tolerancia de las plantas a condiciones salinas (Collins et al., 2008). LaHaye y Epstein (1969) explicaron el papel crucial del $\mathrm{Ca}^{2+}$ en la regulación de la salinidad en las plantas y específicamente en el transporte selectivo o exclusión de $\mathrm{Na}^{+}$y otros iones minerales por las membranas celulares de las plantas. Diversos estudios reportan que la adición de $\mathrm{Ca}^{2+}$ al medio de crecimiento atenúa el estrés por salinidad (Rengel, 1992). Ashraf y Naqvi (1992) reportan que suplementar con $\mathrm{Ca}^{2+}$ en la presencia de salinidad mejoró el crecimiento de Brassica juncea y $B$. napus, pero no de $B$. carinata y $B$. rapa, lo que indica una respuesta diferencial entre los genotipos a la suplementación del $\mathrm{Ca}^{2+}$. Ciertamente, altos niveles de $\mathrm{Na}$ inhiben la absorción de $\mathrm{Ca}^{2+}$ $\mathrm{y} \mathrm{K}^{+}$, que resulta en un antagonismo entre $\mathrm{Na}^{+}$ $\mathrm{y} \mathrm{K}^{+}$(Benlloch et al., 1994). Los cationes $\mathrm{Na}^{+} \mathrm{y}$ $\mathrm{K}^{+}$son conocidos por ser el mayor componente inorgánico del potencial osmótico (Asch et al., 1999). En Brassicas Ashraf y McNeilly (2004) sugieren utilizar una relación alta $\mathrm{K}^{+} / \mathrm{Na}^{+}$en el tejido como criterio para tolerancia a salinidad.

La concentración de magnesio en tejido foliar de brócoli fue creciente en los primeros $20 \mathrm{ddt}$
$(0,54 \%)$, descendiendo hasta los $52 \mathrm{ddt}(0,11 \%)$ y aumentando ligeramente hasta los $61 \mathrm{ddt}(0,14 \%)$. Por lo tanto, en los primeros $33 \mathrm{ddt}$ la concentración total de $\mathrm{Mg}^{2+}$ se ubica dentro de los rangos óptimos de suficiencia (0,25-0,5\%) (Castellanos, 1998). En los días posteriores, se presentó en un rango de deficiencia hasta la cosecha (figura 1E). En repollo, la concentración de $\mathrm{Mg}^{2+}$ (figura 2E) fue alta a los 19 ddt $(0,5 \%)$ y disminuyó hasta los 68 ddt (0,25\%). En general su concentración estuvo en los rangos óptimos de suficiencia (0,20,7\%) sugeridos por Marambio (2004). Ruiz et al. (1997) encontraron que la salinidad por $\mathrm{NaCl}$ redujo la concentración foliar de $\mathrm{Mg}^{2+}$ en cítricos. $\mathrm{Hu}$ y Schmidhalter (1997) demostraron que la concentración de $\mathrm{Mg}^{2+}$ en hojas de trigo se redujo, pero podría ser aumentado para un nivel de salinidad dado, por incrementos en la concentración de $\mathrm{Mg}^{2+}$ en la solución nutritiva.

El boro es un elemento esencial requerido para el crecimiento normal de las plantas superiores. La deficiencia de $B$ es un problema extendido en muchos cultivos agrícolas (Shorrocks, 1997). En el tejido foliar de brócoli, la concentración de B (figura 1F) fue alta a los $19 \mathrm{ddt}\left(25,91 \mathrm{mg} \mathrm{kg}^{-1}\right)$, se redujo levemente hasta los $40 \mathrm{ddt}(17,38 \mathrm{mg}$ $\mathrm{kg}^{-1}$ ) y aumentó hasta los $61 \mathrm{ddt}$, obteniendo una concentración de $25,5 \mathrm{mg} \mathrm{kg}^{-1}$. Los cambios en las concentraciones de $\mathrm{B}$ pueden estar relacionados con los altos requerimientos de este nutriente por las Brassicas en general. Estudios previos muestran que la deficiencia de B disminuye la capacidad fotosintética de las plantas (Kastori et al., 1995; Zhao y Oosterhuis, 2002, 2003). En repollo (figura 2F), a los 19 ddt la concentración de $\mathrm{B}$ fue de 19,14 mg kg-1 , aumentó hasta los 47 ddt (28,97 $\left.\mathrm{mg} \mathrm{kg}^{-1}\right)$ y disminuyó hasta los $68 \mathrm{ddt}(23,83 \mathrm{mg}$ $\mathrm{kg}^{-1}$ ). La deficiencia de B es un principal problema que impide el crecimiento del cultivo y generalmente conduce al rápido cese de la elongación de la raíz, reduce la expansión foliar, principalmente debido a la reducción en la expansión celular (Dell y Huang, 1997; Marschner, 2002). 
Finalmente, la salinidad presente en el suelo y en el agua de riego disminuyeron en brócoli la concentración de $\mathrm{N}, \mathrm{P}, \mathrm{Ca}^{2+} \mathrm{y} \mathrm{Mg}^{2+}$ en el tejido foliar en la etapa intermedia y al final del ciclo del cultivo, coincidiendo con los resultados obtenidos por Cuartero et al. (1992) y Pérez-Alfocea et al. (1996). En tomate, en el cual la salinidad
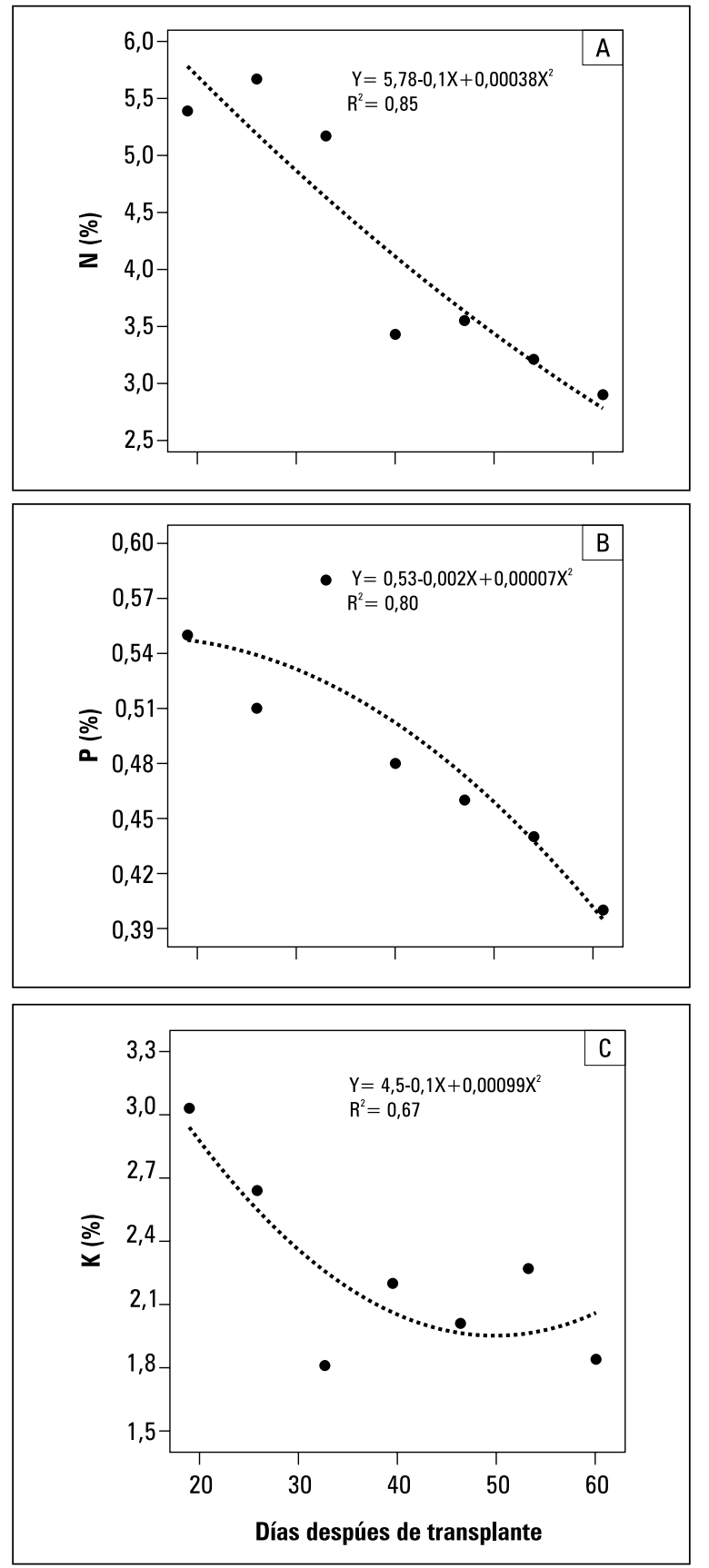

disminuyó en las hojas concentraciones de $\mathrm{K}^{+}$, $\mathrm{Ca}^{2+}, \mathrm{Mg}^{2+}$ y $\mathrm{NO}_{3}^{-}$, las plantas pueden tomar más $\mathrm{K}^{+}, \mathrm{Ca}^{2+}, \mathrm{Mg}^{2+}$ y $\mathrm{NO}^{3-}$ desde el medio si las relaciones $\mathrm{Na}^{+} / \mathrm{K}^{+}, \mathrm{Na}^{+} / \mathrm{Ca}^{2+}, \mathrm{Na}^{+} / \mathrm{Mg}^{2+}$ y el equilibrio de los demás nutrientes son similares al tratamiento control; esto es más evidente en el desarrollo activo de hojas jóvenes.
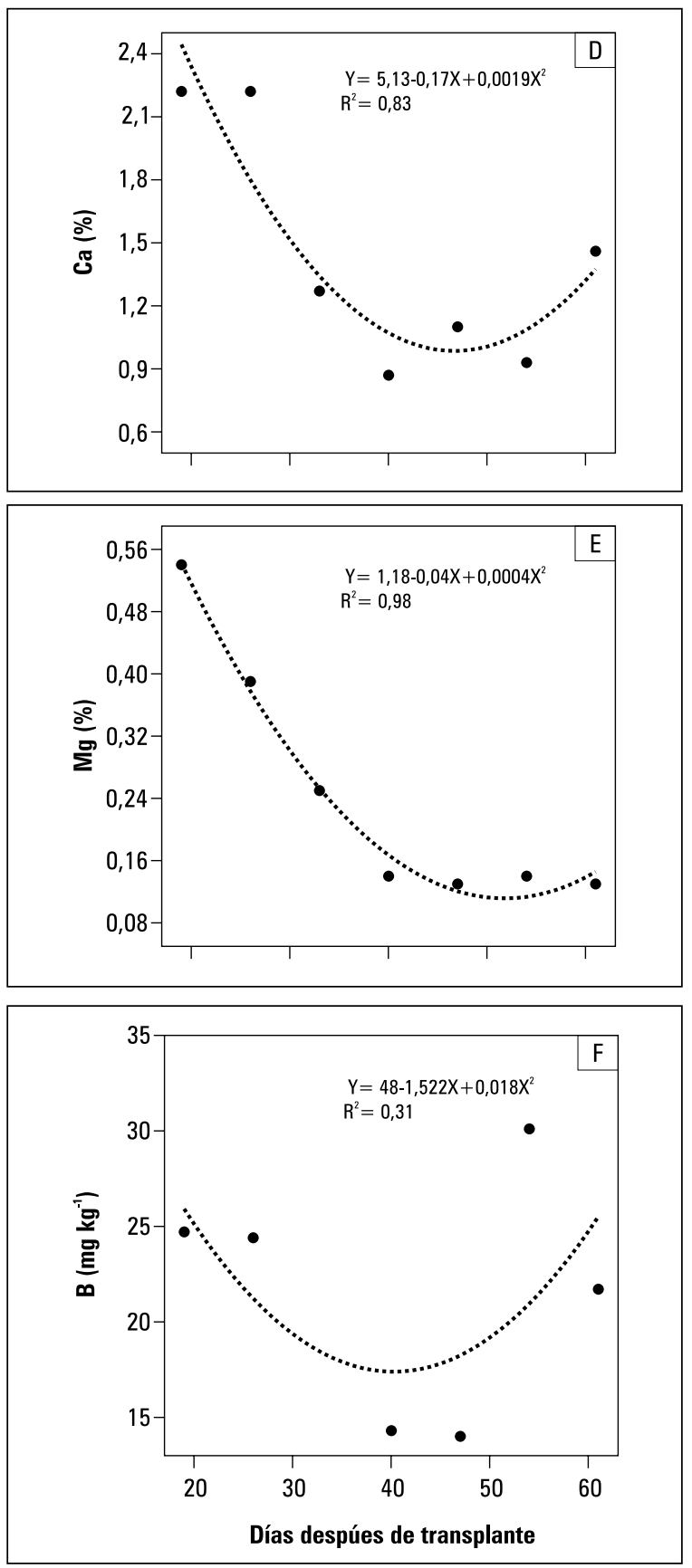

Figura 1. Concentraciones de los nutrientes minerales en tejido foliar en brócoli. A) nitrógeno; B) fósforo; C) potasio; D) calcio; E) magnesio; F) boro. 

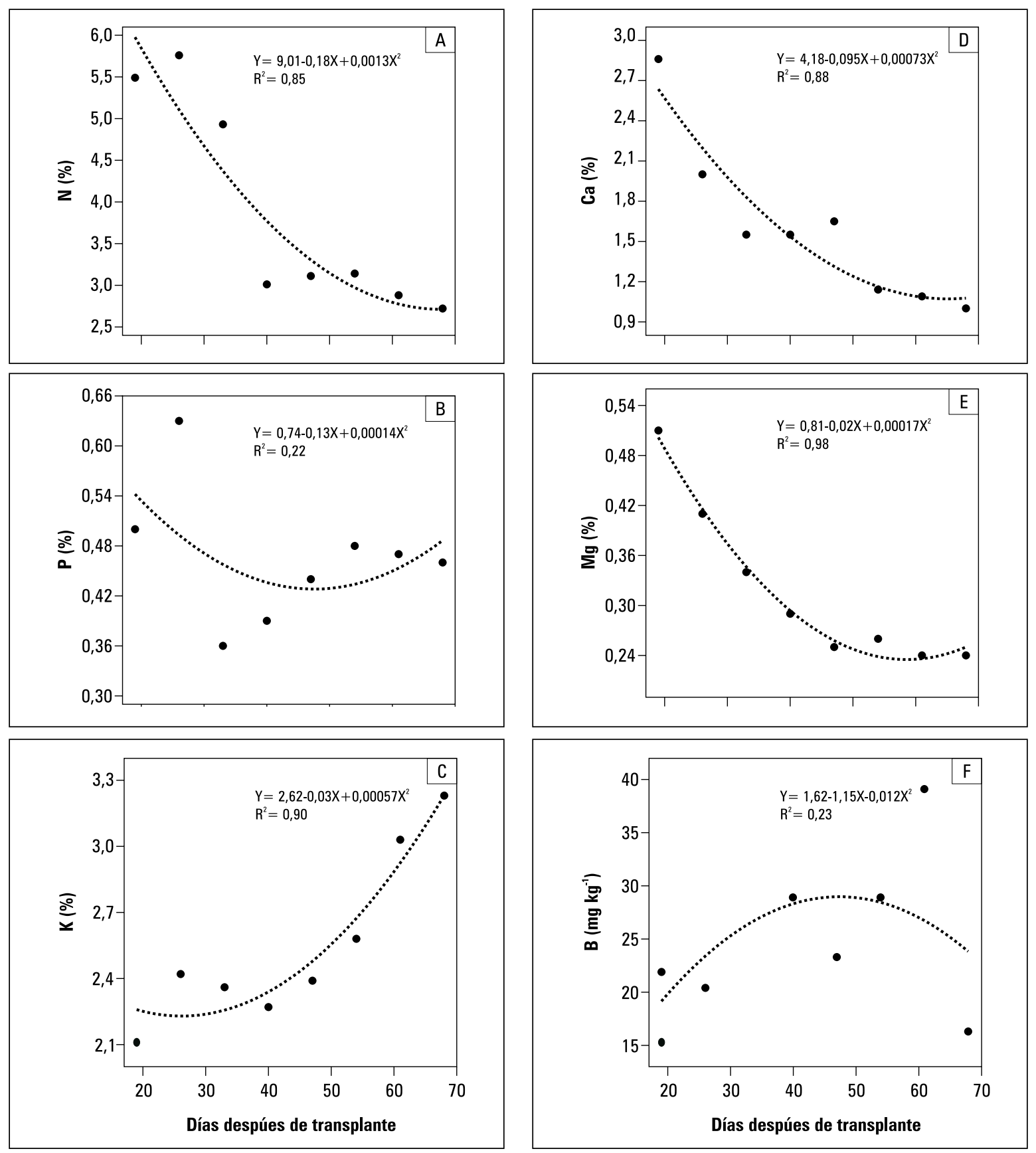

Figura 2. Concentraciones de los nutrientes minerales en tejido foliar en repollo. A) nitrógeno; B) fósforo; C) potasio; D) calcio; E) magnesio; F) boro.

\section{CONCLUSIONES}

- La presencia de sales tanto en el suelo como en el agua de riego influyó en la absorción, transporte y distribución de los elementos minerales en brócoli y repollo.
- En brócoli, las concentraciones de N, P, Ca y $\mathrm{Mg}$ en tejido foliar estuvieron en los rangos adecuados los primeros $51 \mathrm{ddt}$ y posteriormente estuvieron en deficiencia. 
- En repollo, los contenidos de P, Ca y Mg en tejido foliar se presentaron en los rangos adecuados, por ende en esta Brassica se observó cierto nivel de tolerancia a la salinidad, la cual se manifestó en un buen estatus de estos nutrientes.
- En todo el ciclo productivo del brócoli y repollo, se observó deficiencia de K en tejido foliar, mostrando que la salinidad incidió en la disponibilidad de este elemento en la solución del suelo y en la absorción por las plantas.

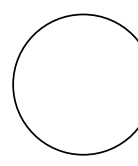

\section{REFERENCIAS BIBLIOGRÁFICAS}

Asch, F.; M. Dingkuhn; C. Wittstock y K. Doerffling. 1999. Sodium and potassium uptake of rice panicles as affected by salinity and season in relation to yield and yield components. Plant Soil 207, 133145.

Ashraf, M. y M.I. Naqvi. 1992. Growth and ion uptake of 4 Brassica species as affected by $\mathrm{Na}^{+} / \mathrm{Ca}^{2+}$ ratio in saline sand culture. Z. Pflanzenernähr. Bodenkd. $155,101-108$.

Ashraf, M. 2004. Some important physiological selection criteria for salt tolerance in plants. Department of Botany, University of Agriculture, Islamabad, Pakistan.

Ashraf, M. y T. McNeilly. 2004. Salinity tolerance in Brassica oilseeds. Crit. Rev. Plant Sci. 23, 157-174.

Asohofrucol. 2007. Brócoli y repollo. En: http://frutasyhortalizas.com.co; consulta: 15 de diciembre de 2007.

Azcón-Bieto, J. y M. Talón. 2008. Fundamentos de fisiología vegetal. 2 da ed. McGraw-Hill Interamericana, Madrid.

Benlloch, M.; M.A. Ojeda; J. Ramos y A. RodríguezNavarro. 1994. Salt sensitivity and low discrimination between potassium and sodium in bean plants. Plant Soil 166, 117-23.

Botella, M.A.; V. Martínez; M. Nieves y A. Cerdá. 1997. Effect of salinity on the growth and nitrogen uptake by wheat seedlings, J. Plant Nutr. 20, 793804.

Castellanos, J.Z. 1998. El seguimiento de la nutrición del cultivo de brócoli en los sistemas de fertirrigación. Instituto Nacional de Investigaciones Forestales y Agropecuarias. En: http://www.ppi-far.org; consulta: 10 de diciembre de 2006 .

CCI. 2006. Entorno nacional. pp. 41-44. En: Plan hortícola nacional. Corporación Colombia Internacional, Bogotá.
Chow, W.S.; M.C. Ball y J.M. Anderson. 1990. Growth and photosynthetic responses of spinach to salinity: Implications of $\mathrm{K}$ nutrition for salt tolerance. Aust. J. Plant Physiol. 17, 563-578.

Collins, R.P.; P.J.C. Harris; M.J. Bateman y J. Henderson. 2008. Effect of calcium and potassium nutrition on yield, ion content, and salt tolerance of Brassica campestris (rapa). J. Plant Nutr. 31, 1461-1481.

Cramer, M.D.; A. Schierholt; Y.Z. Wang y S.H. Lips. 1995. The influence of salinity on the utilization of root anaplerotic carbon and nitrogen metabolism in tomato seedlings. J. Exp. Bot. 46, 1569-1577.

Cuartero, J.; A.R. Yeo y T.J. Flowers. 1992. Selection of donors for salt-tolerance in tomato using physiological traits. New Phytol. 121, 63-69.

Dell, B. y L.B. Huang. 1997. Physiological response of plants to low boron. Plant Soil 193, 103-120.

Feigin, A. 1985. Fertilization management of crops irrigated with saline water. Plant Soil 89, 285-299.

Graifenberg, A.; L. Botrini; L. Giustiniani y M. Lipucci Di Paola. 1996. Salinity affects growth, yield and elemental concentration of fennel. HortScience 31, 1131-1134.

Grattan, S. y C. Grieve. 1999. Salinity-mineral nutrient relations in horticultural crops. Scientia Hort. 78, 127-157.

Greenway, H. y R. Munns. 1980. Mechanisms of salt tolerance in non-halophytes. Annu. Rev. Plant Physiol. 31, 149-190.

Hu, Y. y U. Schmidhalter. 1997. Interactive effects of salinity and macronutrient level on wheat. II. Composition. J. Plant Nutr. 20, 1169-1182.

Hu, Y. y U. Schmidhalter. 2005. Drought and salinity: a comparison of their effects on mineral nutrition of plants. J. Plant Nutr. Soil Sci. 168, 541-549. 
ICA. 1989. El análisis de suelos, plantas y aguas para riego. Manual de Asistencia Técnica No. 47. Bogotá.

IGAC. 1990. Métodos analíticos del Laboratorio de Suelos. 5 a ed. Bogotá.

LaHaye, P.A. y E. Epstein. 1969. Salt toleration by plants: Enhancement with calcium. Science 166, 395-396.

López, M.V. y S.M.E. Satti. 1996. Calcium and potassium-enhanced growth and yield of tomato under sodium chloride stress. Plant Sci. 114, 19-27.

Kastori, R., M. Plesnicar, D. Pankovic y Z. Sakac. 1995. Photosynthesis, chlorophyll fluorescence and soluble carbohydrates in sunflower leaves as affected by boron deficiency. J. Plant Nutr. 18, 1751-1763.

Marambio, C.C. 2004. Curvas de acumulación de materia seca y nutrientes en cultivares de repollo de Bruselas. Tesis de Doctorado. Facultad de Agronomía, Universidad Católica de Chile, Santiago.

Marschner, H. 2002. Mineral nutrition of higher plants. Academic Press, London.

Munns, R. 2003. Comparative physiology of salt and water stress. Plant Cell Environ. 25, 239-250.

Papadopoulous, I. y V.V. Rendig. 1983. Interactive effects of salinity and nitrogen on growth and yield of tomato plants. Plant Soil 73, 47-57.

Patel, A.D. y A.N. Pandey. 2007. Effect of soil salinity on growth, water status and nutrient accumulation in seedlings of Cassia Montana (Fabaceae). J. Arid Environ. 70, 174-182.

Pérez-Alfocea, F.; M.E. Balibrea; A. Santa Cruz y M.T Estaño. 1996. Agronomical and physiological characterization of salinity tolerance in a commercial tomato hybrid. Plant Soil 180, 251-257.

Peuke, A.D., J. Glaab, W.M. Kaiser y W.D. Jeschke. 1996. The uptake and flow of $\mathrm{C}, \mathrm{N}$ and ions between roots and shoots in Ricinus communis L. IV. Flow and metabolism of inorganic nitrogen and malate depending on nitrogen nutrition and salt treatment. J. Exp. Bot. 47, 377-385.
Rengel, Z. 1992. The role of calcium in salt toxicity. Plant Cell Environ. 15, 625-632.

Salisbury, F. y C.W. Ross. 1994. Fisiología vegetal. Grupo Editorial Iberoamericana, México.

Ruiz, D.; V. Martínez y A. Cerda. 1997. Citrus response to salinity: growth and nutrient uptake. Tree Physiol. 17, 141-150.

Sharpley, A.N., J.J. Meisinger, J.F. Power y D.L. Suarez. 1992. Root extraction of nutrients associated with long-term soil management. Adv. Soil Sci. 19, 151217.

Shorrocks, V.M. 1997. The occurrence and correction of boron deficiency. Plant Soil 193, 121-48.

Semillas Arroyave. 2007. Repollo Delus. En: http:// www.semillasarroyave.com; consulta: 11 dediciembre de 2007.

Sen, D.N.; P.K. Kasera y S. Mohammad. 2002. Biology and physiology of saline plants. pp. 563-580. En: Pessaraki, $M$ (ed.). Handbook of plant and crop physiology. $2^{\text {nd }}$ ed. Marcel Dekker, New York, NY.

Song, J.Q. y H. Fujiyama. 1996. Difference in response of rice and tomato subjected to sodium salinization to the addition of calcium. Soil Sci. Plant Nutr. 42, 503-510.

USDA. 2006. Key to soil taxonomy by soil survey staff. United States Government Printing Office, Washington.

Volkmar, K.M.; Y. Hu y H. Steppuhn. 1998. Physiological responses of plants to salinity: a review. Can. J. Plant Sci. 78, 19-27.

Zhao, D. y D.M. Oosterhuis. 2002. Cotton carbon exchange, nonstructural carbohydrates, and boron distribution in tissues during development of boron deficiency. Field Crops Res. 78, 75-77.

Zhao, D. y D.M. Oosterhuis. 2003. Cotton growth and physiological responses to boron deficiency. J. Plant Nutr. 26, 855-867. 